\title{
The capacity of human memory: Is there any limit to human memory?
}

\author{
Ehsan Namaziandost ${ }^{1}$, Meisam Ziafar ${ }^{2}$ \\ 1,2Department of English, Islamic Azad University, Iran \\ 1e.namazi75@yahoo.com, 2meisam-ziafar@iauahvaz.ac.ir \\ *) correspondence: e.namazi75@yahoo.com
}

\begin{abstract}
There exist some estimates of the capacity of human memory. Recent studies have proven the fact that Long Term Memory is subject to constant reconfigurations mostly at lower levels of neural clusters. There is no consensus on one definition for the capacity of memory. As far as retrieval of items present in memory is not the concern, it is reasonable to refrain from putting limits on capacity of human memory; otherwise, one must accept a number game which renders no fixed definite final estimation. Recently such capacity is defined as the amount of interference created by the item which must remain active in the memory.
\end{abstract}

Keywords: human memory; long term memory; interference; capacity

\section{INTRODUCTION}

The human brain consists of about one billion neurons. Each neuron forms about 1,000 connections to other neurons, amounting to more than a trillion connections. "If each neuron could only help store a single memory, running out of space would be a problem. You might have only a few gigabytes of storage space, similar to the space in an iPod or a USB flash drive" (Chen, Hsieh, \& Kinshuk, 2008, p. 8). Yet neurons combine so that each one helps with many memories at a time, exponentially increasing the brain's memory storage capacity to something closer to around 2.5 petabytes (or a million gigabytes)(Wright \& Fergadiotos, 2012). For comparison, if your brain worked like a digital video recorder in a television, 2.5 petabytes would be enough to hold three million hours of TV shows. You would have to leave the TV running continuously for more than 300 years to use up all that storage. The brain's exact storage capacity for memories is difficult to calculate (Murray, 2012). First, we do not know how to measure the size of a memory. Second, certain memories involve more details and thus take up more space; other memories are forgotten and thus free up space. Additionally, some information is just not worth remembering in the first place. This is good news because our brain can keep up as we seek new experiences over our lifetime. If the human life span were significantly extended, could we fill our brains? I'm not sure (Ivanova \& Hallowell, 2012).

One interesting machine is the human brain. In our mind the complex interactions form our emotions, perceptions, feelings and desires and ultimately make us who we are. Is there any end to what this incredible machine can achieve? Is a certain amount of the human intellect capped? When we project ourselves over, say, a thousand years, we can learn and understand a lot more than we do today? Is there an inherent limit to what our brains can understand? So, if we can imagine how powerful the brain is, let's do some maths. There are around 100 billion neurons in the human brain. While many famous sources say that on average each neuron is fired about 200 times per secondand it's the first number you'll get if you search up on Google — this figure is most likely wrong. Scientists are not exactly sure what the number is because different parts of brain pulse at different rates, but a report proposes a rate of 0.29 per second based on rough calculations (Namaziandost, Hafezian, \& Shafiee, 2018). Every neuron is believed to be connected to some 7,000 other neurons, so that 7,000 other neurons get that information every time a single neuron shoots a signal. If you 
multiply these three numbers, you get 200,000,000,000,000 bits of information transmitted every second inside your brain. That's 200 million - a number too big to visualize. The point is: the brain is a powerful machine.

Despite the general agreement on the fact that there is no limit to the storage capacity of human long-term memory, mostly in the case of well-defined upper bounds (Cherniak, 1983; Galton, 1879, cited in Dudai, 2011), some studies have tried to find the capacity limitations of human memory. Dudai (2011) maintains that if by memory capacity we mean memory that can be stored regardless of whether it is normally retrieved or not it can be asserted that everything we learn is permanently stored in the mind. Dudai (2011) cites the French philosopher Helvetius who contends that:

"The capacity of memory far exceeds the practical needs of a thoughtful human being. There is no one whose memory cannot contain not only all the words of a language, but also an infinity of dates, facts, names, places, and finally a number of objects considerably more than six or seven thousand". (p. 342)

The other premodern attempt in determining the capacity of human memory was done by a Swiss-German psychologist, Haller, who estimated that within 50 years a person may accumulate 1,577,880,000 traces (Burnham, 1889, cited in Dudai, 2011). After presenting some attempts in measuring the amount of memory capacity in humans Dudai (2011) concludes that it cannot be done yet since we still do not know how representations are in the spatial and temporal patterns of neuronal activity. What is more current formal estimates of maximal information in our brain converges on $\square 10^{\wedge} 15-\square 10^{\wedge} 17$ bits $(\square=0.14-2)$.

According to Wang, Liu and Wang (2003) the brain of an adult person contains up to 100 billion neurons, when each neuron is linked to a vast number of other neurons through thousands of synapses. They maintain that despite having such knowledge the capacity of human brain remains a mystery to be discovered, since such estimation highly depends on proper cognitive and mathematical models of the brain. They further maintain that despite the belief that Long Term Memory (LTM) is fixed and static, which is based on observations showing that the capacity of adult brains is already completed and has reached a static state and its growth is impeded, recent discoveries in neuroscience and informatics show that LTM is being constantly reconfigured, mainly at lower levels of the neural clusters. After going through some mathematical and computational algorithms, as a rough estimation of human memory capacity they propose $10^{\wedge} 8432$ bits as a rough estimation of the human memory capacity. The authors further maintain that the brain does not create new neurons to represent new information; rather it sets new synapses between the existing neurons so that new information can be represented.

After all, the important fact to bear in mind is that as Dudai (2011) rightly mentions, memory capacity means different things to different people:

Before embarking on the quest for pertinent data, it is useful to note that "memory capacity" means different things to different people. Theoreticians may construe it as referring to the overall storage capacity of an information-processing machine with the properties of brain. Those who are more biologically oriented may wish to add that the capacity of brains is constrained by deterioration with age and the finite life time of mortals. Experimental psychologists and neuropsychologists may have in mind distinct memory systems and may also ask how much of the capacity of each system is actually used. It is evident that the theoretical limit is larger than the real-life overall capacity, which is larger than the capacity of specific systems, etc. (p. 343)

According to Dudai (2011) other attempts made in order to determine the amounts of human capacity were geared to estimating the maximal amount of information that the human brain is expected to perceive throughout life.

A pioneer in the modern attempts to equate the brain with a machine, John Von Neuman, estimated that the input per nerve cell per second in the human brain is 14 bits, and, concluded that the information accumulated over a life span of 60 years is $2.8 \times 1020$ bits (von Neuman 1958). In the past 20 years both life expectancy and the estimate of the number of neurons in the brain 
increased; therefore, using the same influx estimate one now gets $3.3 \times 1021$ bits per lifetime. (p. 345).

The conclusions drawn by Dudai (2011, pp. 347-348) are as follow:

1- Although the theoretical constructs of mathematicians, physicists, and other practitioners of artificial neural networks vary in their assumptions and rules, the bottom line is that the human brain (or,more accurately, its artificial simplified counterparts) is capable of a storage capacity that can account for the higher-end estimates obtained by either folk or experimental psychology. That is, wewould not be able to astonish the current generation of modelers if we were to confront them with the views of St. Augustine or Hailer; they surely would be able to come up with a model that reconciles with the data. The appreciation for this truly impressive capacity is clearly detected in various treatments of the subject in computer sciences and information theory.

2- Highly simplified number games, suggest that in theory, the human brain may be capable of storing all of the sensory information that it encounters throughout a lifetime.

3-Last, but not at all least: There is a link missing between theory and biology, without which the calculation would indeed remain number games. Even if we were to know how many bits the brain can store, this would not tell us how many memories we have because we do not know, first, how much of the system is engaged in mnemonics and second, most importantly, how specific pieces of information are encoded.

\section{Short-Term Memory (STM) Capacity and Working Memory Capacity (WMC)}

There are various estimates for the storage capacity of short-term memory. Cherniak (1983) considers it to be six meaningful units or chunks such as randomly chosen words. Miller (1956, cited in Dudai, 2011) considers it to be seven plus or minus two items, and more recently Miller has changed the number into the average capacity of about four numbers (Cowan, 2001, cited in Morey, 2011).

According to Baddeley (1996) the concept of working memory is different from that of shortterm memory in two respects. Firstly, the working memory is composed of a number of subsystems rather than being a unitary module, and secondly, it involves an emphasis on the functional role of other cognitive tasks such as learning, reasoning and comprehension, to the extent that functional capacity of working memory (Ricks \& Wiley, 2009) is preferred. The storage component of working memory, according to Morey (2011), is considered as a buffer that permits the manipulation and working on information which comes from various sources like sensory stores or long-term memory. In regard to the capacity of working memory (WMC) there is general agreement that there is limitation on the amount of processable input, although different scholars propose different estimations. For example Conway, Kane, and Engle (2003) correlate it with Spearman's $g$ (general intelligence),Kyllonen\&Christal (1990) equate working memory capacity with reasoning ability, and Baddeley and Hitch (1974, cited in Baddeley, 2003) propose two separate working memory capacities, with one predicting verbal abilities and the other prediction visuospacial ones.

Gordon et al (2002, cited in Fedorenko, Gibson, \& Rohde, 2006) argued that the working memory capacity in language processing should not be determined in terms of the number of items that can be kept active in memory through the process of comprehension, but rather in terms of the amount of interference created by the item which must remain active in the memory. In the case of the capacity of Long Term Memory, Brady, Konkle, Alvarez, and Oliva (2008) also refute the idea that the number of stored items can be used as an estimate of memory capacity, and propose the proper capacity estimate take into account the number of remembered items multiplied by the amount of information present within each item.

\section{CONCLUSION}

Those interested in knowing about how the capacity of human memory is limited should not expect to be presented with more than a number game. Different numbers are achieved by numerous scholars due to their special orientations toward the issue. When by capacity of memory we mean 
those items, which are accumulated and the retrieval of the items already present in the memory is not considered as a criterion in setting limits for memory capacity it is much easier to go into the camp of those who see no point in limiting memory capacity. Despite this disagreement it can be concluded that as Dudai (2011) maintains, current formal estimates of maximal information in our brain converges on $\square 10^{\wedge} 15-\square 10^{\wedge} 17$ bits $(\square=0.14-2)$. Through making peace between theory and biology one is more likely to end in closer estimates of the capacity of human memory and thus to put an end on this number game. The presence of such a number game implies that there is almost agreement on setting limits on the capacity of human memory. The capacity of working memory as an indispensable part of human memory highly correlates with higher order manipulations of items such as reasoning and comprehension. Recent views have a more comprehensive definition for what the capacity of working memory is. They define it as the amount of interference created by the item which must remain active in the memory.

\section{REFERENCES}

Baddeley, A. D. (1996). The fractionation of working memory. Proc. Natl. Acad. Sci., 93, 1346813472.

Baddeley, A. D. (2003). Working memory: Humans. In J. H. Byrne (Ed.), Learning and memory (pp. 672-676). New York: MacMillan.

Brady, T. F., Konkle, T., Alvarez, G. A., \& Oliva, A. (2008). Visual long-term memory has a massive storage capacity for object details. PNAS, 105(38), 14325-14329.

Chen, N.S., Hsieh, S.W., \& Kinshuk . (2008). Effects of short-term memory and content representation type on mobilelanguage learning. Language Learning \& Technology, 12(3), 93-113.

Cherniak, C. (1983). Rationality and the structure of human memory. Synthese, 57, 163-186.

Conway, A. R. A., Kane, M. J., Engle, R. W. (2003). Working memory capacity and its relation to general intelligence. Trends in Cognitive Sciences, 7(12), 547-552.

Dudai, Y. (2011). How big is human memory? Learning \& Memory, 3, 341-365.

Fedorenko, E., Gibson, E., \& Rodhe, D. (2006). The nature of working memory capacity in sentence comprehension: Evidence against domain-specific working memory resources. Journal of Memory and Language, 54, 541-553.

Ivanova, M.V., \& Hallowell, B. (2012). Validity of an eye-tracking method to index working memory in people with and without aphasia. Aphasiology, 26, 556-578.

Kyllonen, P.C., \&Christal, R.E. (1990). Reasoning ability is (little more than) working memory capacity. Intelligence, 14, 389-433.

Morey, R. D. (2011). A Bayesian hierarchical model for the measurement of working memory capacity. Journal of Mathematical Psychology, 55, 8-24.

Murray, L. L. (2012). Attention and other cognitive deficits in aphasia: presence and relation to language and communication measures. American Journal of Speech-Language Pathology, 21, S51-S64.

Namaziandost, E., Hafezian, M., \&Shafiee, S. (2018). Exploring the association among working memory, anxiety and Iranian EFL learners' listening comprehension. Asian-Pacific Journal of Second and Foreign Language Education, 3(20), 1-17. https://doi.org/10.1186/s40862-018-00613.

Ricks, T. R., \& Wiley, J. (2009). The influence of domain-knowledge on the functional capacity of working memory. Journal of Memory and Language, 10, 1-19.

Wang, Y., Liu, D., \& Wang, Y. (2003). Discovering the capacity of human memory. Brain and Mind, 4, 189-198.

Wright, H.H., \&Fergadiotos, G. (2012). Conceptualizing and measuring working memory and itsrelationship to aphasia. Aphasiology, 26, 258-278. 